\title{
Características Físico-Química, Energética e Dimensões das Fibras de Três Espécies Florestais do Semiárido Brasileiro
}

\author{
Juarez Benigno Paes ${ }^{1}$, Carlos Roberto de Lima², Elisabeth de Oliveira², \\ Pedro Nicó de Medeiros Neto ${ }^{3}$
}

\footnotetext{
${ }^{1}$ Departamento de Ciências Florestais e da Madeira, Universidade Federal do Espírito Santo - UFES, Jerônimo Monteiro/ES, Brasil

${ }^{2}$ Unidade Acadêmica de Engenharia Florestal, Universidade Federal de Campina Grande - UFCG, Patos/PB, Brasil

${ }^{3}$ Programa de Pós-graduação em Ciências Florestais, Universidade Federal do Espírito Santo - UFES, Jerônimo Monteiro/ES, Brasil
}

\section{RESUMO}

Esta pesquisa teve como objetivo avaliar as características físico-química, energética e dimensões das fibras das madeiras de angico-vermelho (Anadenanthera colubrina var. cebil), jurema-preta (Mimosa tenuiflora) e jurema-vermelha (Mimosa arenosa), espécies de ocorrência no Semiárido Brasileiro e empregadas para energia. Para tanto, foram colhidas cinco árvores de cada espécie estudada. A caracterização física foi realizada no Laboratório de Tecnologia de Produtos Florestais (LTPF) da Universidade Federal de Campina Grande e a caracterização química e a análise das dimensões das fibras no Laboratório de Papel e Celulose da Universidade Federal de Viçosa. De modo geral, as madeiras ensaiadas tiveram características físicas e dimensões das fibras semelhantes. Quanto às características químicas, a jurema-preta teve maior solubilidade em álcool:tolueno, maior teor de lignina total e menores teores de holocelulose e cinzas do que as demais espécies, tendo três vezes menos cinzas do que a madeira de angico-vermelho e a metade do apresentado pela jurema-vermelha.

Palavras-chave: espécies da caatinga, madeira, energia.

\section{Physical-Chemical, Energetic Characteristics and Fiber Dimensions of Three Forestry Species of Brazilian Semi-Arid Region}

\begin{abstract}
In this study, we aimed to evaluate the physico-chemical, energetic characteristics and fiber dimensions of Anadenanthera colubrina var. cebil, Mimosa tenuiflora and Mimosa arenosa woods, species of Brazilian Semiarid region used for energy. Five trees of each species were logged. The physical characterization of wood was performed in the Forest Products Technology Laboratory (FPTL) of the Federal University of Campina Grande and fiber dimensions and chemical characterization in the Pulp and Paper Laboratory at the Federal University of Viçosa. In general, the woods tested presented similar physical characteristics and fiber dimensions. As for the chemical characteristics, Mimosa tenuiflora showed greater alcohol:toluene solubility and total lignin content, lower holocellulose and ash contents than the other species, presenting three times less ashes than Anadenanthera colubrina var. cebil and half the amount presented by Mimosa arenosa.
\end{abstract}

Keywords: species from caatinga, wood, energy. 


\section{INTRODUÇÃO}

Em função de o Bioma Caatinga ter uma vegetação composta por plantas arbustivas, sua utilização econômica tem se restringido ao emprego como forragem e para fins medicinais, taninos e energia. $\mathrm{O}$ emprego da madeira para fins energéticos é expressivo na região, principalmente por causa dos preços mais elevados dos outros insumos. No entanto, a potencialidade energética das espécies da região ainda é pouco estudada.

As características anatômicas (Burger \& Richter, 1991), a composição química (Sjöström, 1993) e a densidade (Vital, 1984) formam os parâmetros que constituem a base para quaisquer estudos tecnológicos que sejam efetuados com a madeira, permitindo empregá-la corretamente em determinado uso.

A densidade básica da madeira é uma característica complexa, resultante da combinação de fatores genéticos e ambientais. Segundo Panshin \& De Zeeuw (1980), é uma característica dependente da interação entre as propriedades anatômicas e químicas da madeira; portanto, as variações na densidade são provocadas por diferenças nas dimensões celulares e pela variação nos componentes químicos da madeira.

A madeira é composta, basicamente, de celulose (40-45\%), hemiceluloses (15-35\%, nas folhosas, e 20 - 30\%, nas coníferas), lignina (18-25\%, nas folhosas, e 25-35\%, nas coníferas), extrativos (3-8\%) e cinzas (0,4\%) (Sjöström, 1993). A celulose, um polissacarídeo, é o principal componente da parede celular e é constituída por uma cadeia homogênea e não ramificada. As hemiceluloses são polissacarídeos de cadeias ramificadas, mais curtas do que a celulose e heterogêneas, com regiões amorfas e semicristalinas (Lewin \& Goldstein, 1991). A lignina é um composto fenólico amorfo, tridimensional, de composição química complexa, com função adesiva entre as fibras, conferindo dureza e rigidez à parede celular (Pettersen, 1984). A lignina tem fundamental importância na produção de carvão vegetal, pois é o composto que mais contribui para a produção do carvão e do alcatrão insolúvel (Oliveira et al., 1982).

Os extrativos não fazem parte da constituição química da parede celular e incluem elevado número de compostos (resinas, açúcares, taninos e ácidos graxos), os quais influenciam as propriedades tecnológicas da madeira. As cinzas são compostas por cálcio, potássio, magnésio e outros minerais (Sjöström, 1993; Pettersen, 1984).

Em função das premissas levantadas e considerando-se a importância da flora da Caatinga como sustento para a população rural (Xavier et al., 2005; Lima et al., 2006), esta pesquisa teve como objetivo avaliar as características físico-químicas, energética e as dimensões das fibras das madeiras de angico-vermelho (Anadenanthera colubrina var. cebil), jurema-preta (Mimosa tenuiflora) e juremavermelha (Mimosa arenosa).

\section{MATERIAL E MÉTODOS}

\subsection{Procedência, coleta e amostragem da madeira}

Para as análises das características físicas, químicas, energéticas e das dimensões das fibras das espécies, foram selecionadas aleatoriamente cinco árvores de cada uma das seguintes espécies: angicovermelho [Anadenanthera colubrina (Vell.) Brenan. var. cebil (Gris.) Alts.], jurema-preta [Mimosa tenuiflora (Willd.) Poir.] e jurema-vermelha [Mimosa arenosa (Willd.) Poir.], que tinham boa fitossanidade, na Fazenda Lameirão, Universidade Federal de Campina Grande (UFCG), localizada no Município de Santa Terezinha, Estado da Paraíba, com Latitude $\mathrm{S} 7^{\circ}$, Longitude W $37^{\circ} 04^{\prime}$, Altitude 240 metros e clima, segundo a classificação de Köppen, semiárido do tipo Aw', quente úmido, com chuvas de verão e outono, média anual das precipitações pluviométricas em torno de $600 \mathrm{~mm}$, distante $385 \mathrm{~km}$ de João Pessoa, Capital do Estado. Os solos predominantes são do tipo litólico eutrófico com afloramentos rochosos e a topografia possui ondulação com presença de pequenas serras (SUDEMA, 2004; IBGE, 2008).

De cada árvore abatida, foram retirados discos de $\pm 2,5 \mathrm{~cm}$ de espessura a 0 (base), 25, 50, $75 \mathrm{e}$ $100 \%$ da altura comercial do tronco, considerada até $3 \mathrm{~cm}$ de diâmetro. Os discos foram subdivididos em quatro seções diametralmente opostas. A primeira seção foi utilizada para as análises anatômicas e químicas; a segunda e a quarta, para a determinação 
da densidade básica, e a terceira reservada para necessidades posteriores.

\subsection{Caracterizações anatômicas, físico- químicas e energéticas das madeiras}

As análises físico-químicas das madeiras foram realizadas nos Laboratórios de Tecnologia de Produtos Florestais (LTPF) da Unidade Acadêmica de Engenharia Florestal (UAEF), do Centro de Saúde e Tecnologia Rural (CSTR) da UFCG, Campus de Patos, em Patos, Paraíba. As análises anatômicas e as leituras do teor de lignina solúvel das madeiras ocorreram no Laboratório de Papel e Celulose da Universidade Federal de Viçosa (UFV), Viçosa, Minas Gerais.

A densidade básica foi determinada de acordo com o método de imersão em água, descrito por Vital (1984), e a análise anatômica das espécies, com base nas dimensões das fibras (comprimento, largura, espessura de parede e diâmetro de lume). Para tanto, foram confeccionados palitos da seção destinada para as análises anatômicas e químicas. Os palitos foram tratados com solução macerante, de acordo com o método de Nicholls, descrito por Ramalho (1987). As medições das dimensões das fibras foram realizadas em um microscópio óptico acoplado a um projetor, sendo a imagem gerada capturada e analisada pelo programa 'Pro-Imagem 3.2', ao serem realizadas medidas em cem fibras por árvore. A espessura média das paredes das fibras foi considerada como a metade da diferença entre a largura da fibra e o diâmetro do lume.

As amostras destinadas às análises químicas foram secas ao ar e transformadas em serragem em moinho tipo Willey, classificadas em peneiras de 40 e 60 'mesh', e condicionadas a $65 \pm 5 \%$ de umidade relativa e a $25 \pm 2{ }^{\circ} \mathrm{C}$. As análises químicas foram realizadas em duplicata e, para cada árvore, foram efetuadas determinações quantitativas dos teores de holocelulose, lignina (solúvel e insolúvel), extrativos totais, cinzas e poder calorífico superior.

A determinação do teor de extrativos na madeira (solubilidade em álcool:tolueno) foi efetuada segundo a M 3/89 da Associação Brasileira Técnica de Celulose e Papel - ABTCP (1974). O teor de lignina foi determinado conforme a metodologia descrita por Gomide \& Demuner (1986), sendo realizada a leitura do filtrado restante da análise em espectrofotômetro para determinação da lignina solúvel em ácido. O teor de lignina total foi o resultado da soma da lignina residual mais a lignina solúvel. O teor de holocelulose foi obtido por diferença [\% holocelulose $=100-$ (teor de extrativos + teor de lignina + cinzas na madeira)]. A determinação do teor de cinzas ou minerais da madeira foi efetuada segundo a M-11/77 (ABTCP, 1974).

$\mathrm{O}$ poder calorífico superior da madeira foi determinado por meio de calorímetro adiabático, de acordo com a Norma Brasileira Regulamentadora - NBR 8633 da Associação Brasileira de Normas Técnicas - ABNT (1983), com madeira absolutamente seca. As análises químicas foram realizadas em duplicata.

\subsection{Análise e avaliação dos resultados}

Para a avaliação dos resultados das características das madeiras estudadas, foi utilizado um delineamento inteiramente casualizado, em que foi avaliado o efeito das espécies em cada característica, contendo cinco repetições por espécie.

Os dados em porcentagem foram transformados em arcsen [raiz quadrada (valor em porcentagem/100)]. Essa transformação dos dados, sugerida por Steel \& Torrie (1980), foi necessária para homogeneizar as variâncias.

\section{RESULTADOS E DISCUSSÃO}

\subsection{Dimensões das fibras das espécies estudadas}

Na Tabela 1, consta o resumo das análises de variâncias para as dimensões das fibras das espécies estudadas. Das características anatômicas analisadas, apenas o diâmetro do lume das fibras teve diferenças significativas entre as espécies. Esse fato comprova que as madeiras eram bem semelhantes, pois duas delas (jurema-preta e jurema-vermelha) pertencem ao gênero botânico Mimosa e as três espécies, à família Mimosaceae.

As médias dos valores obtidos foram comparadas pelo teste de Tukey a $5 \%$ de probabilidade e constam da Tabela 2. Dentre as médias das características 
Tabela 1. Resumo das análises de variância para os valores de comprimento e largura de fibras, diâmetro de lume e espessura da parede das fibras das madeiras estudadas.

Table 1. Summary of variance analysis to values of fiber length, width, lumen diameter and wall thickness of woods studied.

\begin{tabular}{cccccc} 
Fontes de & Graus de & \multicolumn{4}{c}{ Quadrados Médios } \\
\cline { 3 - 6 } Variação & Liberdade & $\begin{array}{c}\text { Comprimento } \\
(\mathbf{m m})\end{array}$ & $\begin{array}{c}\text { Largura } \\
(\boldsymbol{\mu m})\end{array}$ & $\begin{array}{c}\text { Diâmetro do } \\
\text { Lume }(\boldsymbol{\mu m})\end{array}$ & $\begin{array}{c}\text { Espessura de } \\
\text { Parede }(\boldsymbol{\mu m})\end{array}$ \\
\hline Espécie & 2 & $0,62 \times 10^{-2} \mathrm{~ns}$ & $4,72 \mathrm{~ns}$ & $7,65^{* *}$ & $0,13 \mathrm{~ns}$ \\
\hline Resíduo & 12 & $0,22 \times 10^{-2}$ & 1,63 & 1,01 & 0,20 \\
\hline Coeficiente de Variação $(\%)$ & 6,48 & 7,83 & 17,09 & 8,48 \\
\hline
\end{tabular}

** significativo a $1 \%(\mathrm{p} \leq 0,01)$; ns: não significativo a $5 \%(\mathrm{p}>0,05)$.

Tabela 2. Valores médios para comprimento, largura, diâmetro de lume e espessura da parede das fibras das madeiras estudadas.

Table 2. Mean values of length, width and lumen diameter and wall thickness of the fibers from studied woods.

\begin{tabular}{lcccc}
\multicolumn{1}{c}{ Espécies } & $\begin{array}{c}\text { Comprimento } \\
(\mathbf{m m})^{*}\end{array}$ & $\begin{array}{c}\text { Largura } \\
(\mu \mathbf{m}) *\end{array}$ & $\begin{array}{c}\text { Diâmetro do } \\
\text { Lume }(\mu \mathbf{m})\end{array}$ & $\begin{array}{c}\text { Espessura da } \\
\text { Parede }(\mu \mathbf{m}) *\end{array}$ \\
Angico-vermelho & 0,76 & 17,33 & $7,05 \mathrm{a}$ & 5,14 \\
Jurema-preta & 0,72 & 16,23 & $6,01 \mathrm{ab}$ & 5,11 \\
Jurema-vermelha & 0,69 & 15,39 & $4,59 \mathrm{~b}$ & 5,40 \\
\hline
\end{tabular}

As médias seguidas por uma mesma letra, em cada coluna, não diferem estatisticamente (Tukey, p > 0,05). ${ }^{*}$ não significativo (F, $\mathrm{p}>0,05)$.

analisadas, apenas o diâmetro do lume teve diferenças significativas. Neste caso, o angicovermelho teve o maior diâmetro de lume e a juremavermelha, o menor, tendo a jurema-preta um valor intermediário.

\subsection{Características físico-químicas e energéticas das madeiras}

A madeira de angico-vermelho teve uma densidade básica de 0,78 \pm 0,02 g.cm ${ }^{-3}$; a de jurema-preta, de 0,88 $\pm 0,01$ g.cm ${ }^{-3}$, e a de juremavermelha, de $0,83 \pm 0,06 \mathrm{~g} . \mathrm{cm}^{-3}$, com coeficiente de variação de 3,08; 1,00, e 7,69\%, respectivamente. Os valores das densidades básicas e dos poderes caloríficos superiores das madeiras foram analisados estatisticamente e estão apresentados na Tabela 3.

As madeiras das espécies ensaiadas tiveram densidades diferentes e poderes caloríficos superiores semelhantes. As médias dos valores obtidos, quando necessário, foram comparadas pelo teste de Tukey a $5 \%$ de probabilidade e constam da Tabela 4 .

A madeira de jurema-preta teve densidade básica superior à do angico-vermelho, enquanto a madeira de jurema-vermelha teve valores intermediários entre as madeiras ensaiadas. As diferenças entre as
Tabela 3. Resumo das análises de variância para os valores de densidade básica e poder calorífico superior das madeiras estudadas.

Table 3. Summary of variance analysis to basic density and higher calorific value of studied woods.

\begin{tabular}{|c|c|c|c|}
\hline \multirow[b]{2}{*}{$\begin{array}{l}\text { Fontes de } \\
\text { Variação }\end{array}$} & \multirow[b]{2}{*}{$\begin{array}{c}\text { Graus de } \\
\text { Liberdade }\end{array}$} & \multicolumn{2}{|c|}{ Quadrados Médios } \\
\hline & & $\begin{array}{c}\text { Densidade } \\
\text { Básica } \\
\left(\mathrm{g} \cdot \mathrm{cm}^{-3}\right)\end{array}$ & $\begin{array}{c}\text { Poder } \\
\text { Calorífico } \\
\text { Superior } \\
\left(\mathrm{kcal}^{\mathrm{k}} \mathrm{kg}^{-1}\right)\end{array}$ \\
\hline Espécie & 2 & $0,14 \times 10^{-1 \star *}$ & $3.425,20 \mathrm{~ns}$ \\
\hline Resíduo & 12 & $0,16 \times 10^{-2}$ & $2.587,97$ \\
\hline Coeficiente $\mathrm{d}$ & Variação (\%) & 4,80 & 1,13 \\
\hline
\end{tabular}

Tabela 4. Valores médios de densidade básica e poder calorífico superior das madeiras estudadas.

Table 4. Mean values of wood density and higher calorific value of studied woods.

\begin{tabular}{|c|c|c|}
\hline Espécies & $\begin{array}{c}\text { Densidade } \\
\text { Básica } \\
\left(\mathrm{g} \cdot \mathrm{cm}^{-3}\right)\end{array}$ & 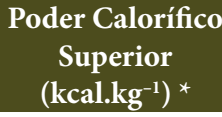 \\
\hline Angico-vermelho & $0,78 \mathrm{~b}$ & $4.499,80$ \\
\hline Jurema-preta & $0,88 \mathrm{a}$ & $4.501,59$ \\
\hline Jurema-vermelha & $0,83 \mathrm{ab}$ & $4.546,00$ \\
\hline
\end{tabular}

As médias seguidas por uma mesma letra, em cada coluna, não diferem estatisticamente (Tukey, p > 0,05). * não significativo (F, $\mathrm{p}>0,05)$. 
Tabela 5. Resumo das análises de variância para os valores de solubilidade em álcool:tolueno, teores de lignina, de holocelulose e de cinzas das madeiras estudadas. Dados transformados em arcsen [raiz quadrada (valores em porcentagem/100)].

Table 5. Summary of variance analysis to values of alcohol:toluene solubility, lignin, holocellulose, and ash content of studied woods. Transformed data in arcsen [square root (values in percentage/100)].

\begin{tabular}{|c|c|c|c|c|c|}
\hline \multirow{2}{*}{$\begin{array}{l}\text { Fontes de } \\
\text { Variação }\end{array}$} & \multirow{2}{*}{$\begin{array}{l}\text { Graus de } \\
\text { Liberdade }\end{array}$} & \multicolumn{4}{|c|}{ Quadrados Médios } \\
\hline & & $\begin{array}{l}\text { Solubilidade } \\
\text { Álcool:Tolueno }\end{array}$ & Lignina & Holocelulose & Cinzas \\
\hline Espécie & 2 & $0,14 \times 10^{-1 \star \star}$ & $0,13 \times 10^{-3 * *}$ & $0,14 \times 10^{-1 \star *}$ & $0,56 \times 10^{-2 * *}$ \\
\hline Resíduo & 12 & $0,42 \times 10^{-4}$ & $0,44 \times 10^{-6}$ & $0,36 \times 10^{-3}$ & $0,16 \times 10^{-3}$ \\
\hline \multicolumn{2}{|c|}{ Coeficiente de Variação (\%) } & 2,47 & 1,31 & 1,99 & 11,08 \\
\hline
\end{tabular}

${ }^{* *}$ significativo a $1 \%(\mathrm{p} \leq 0,01)$.

Tabela 6. Valores médios de solubilidade em álcool:tolueno, teor de lignina, de holocelulose e de cinzas das madeiras estudadas.

Table 6. Mean values of alcohol:toluene solubility, lignin, holocellulose, and ash content of studied woods.

\begin{tabular}{ccccc} 
Espécies & $\begin{array}{c}\text { Solubilidade } \\
\text { Álcool:Tolueno (\%) }\end{array}$ & $\begin{array}{c}\text { Lignina } \\
(\%)\end{array}$ & $\begin{array}{c}\text { Holocelulose } \\
(\%)\end{array}$ & $\begin{array}{c}\text { Cinzas } \\
(\%)\end{array}$ \\
\hline Angico-vermelho & $4,85 \mathrm{c}$ & $24,85 \mathrm{~b}$ & $68,20 \mathrm{a}$ & $2,10 \mathrm{a}$ \\
\hline Jurema-preta & $9,96 \mathrm{a}$ & $29,14 \mathrm{a}$ & $60,28 \mathrm{~b}$ & $0,62 \mathrm{c}$ \\
\hline Jurema-vermelha & $5,80 \mathrm{~b}$ & $23,32 \mathrm{~b}$ & $69,51 \mathrm{a}$ & $1,37 \mathrm{~b}$ \\
\hline
\end{tabular}

As médias seguidas por uma mesma letra, em cada coluna, não diferem estatisticamente (Tukey, p > 0,05).

densidades das espécies não foram suficientes para causar variações significativas no poder calorífico superior das madeiras (Tabela 3). Os poderes caloríficos estão em conformidade com os valores obtidos por Oliveira et al. (2006), para a madeira de jurema-preta.

$\mathrm{Na}$ Tabela 5, consta o resumo das análises de variância para as características químicas das madeiras, tendo sido avaliados, além da solubilidade em álcool:tolueno, os teores de lignina, de holocelulose e de cinzas nas madeiras.

Todas as características avaliadas apresentaram diferenças significativas para as espécies estudadas. As médias de cada característica foram comparadas pelo teste de Tukey a $5 \%$ de probabilidade e os resultados apresentados na Tabela 6 .

A madeira de jurema-preta possui a maior solubilidade em álcool:tolueno, quando comparada às demais espécies, e o angico-vermelho, a menor. Quanto ao teor de lignina, a madeira de jurema-preta teve um teor superior ao do angico e da juremavermelha, tendo, por consequência, um menor teor de holocelulose.

O teor de cinzas da madeira de jurema-preta foi inferior ao das demais espécies, sendo três vezes menor do que o do angico-vermelho e a metade do apresentado pela jurema-vermelha. Esta característica da madeira é importante, pois serão necessárias menos limpezas em fornalhas e em outros sistemas onde a madeira é utilizada como fonte de energia (Oliveira, 2003).

Em função do maior teor de lignina e do menor teor de cinzas, a jurema-preta possui boas características energéticas, com potencialidade para ser empregada para fins siderúrgicos.

\section{CONCLUSÕES}

As densidades básicas das madeiras apresentaram pouca variação entre as espécies estudadas. A densidade básica da jurema-preta foi superior à do angico-vermelho. As variações nas densidades básicas não foram suficientes para influenciar os poderes caloríficos superiores das madeiras estudadas.

As madeiras das espécies ensaiadas tiverem comprimento, largura e espessura da parede das fibras semelhantes. O diâmetro do lume das fibras do angico-vermelho foi superior ao da jurema-preta.

A jurema-preta teve maior solubilidade em álcool:tolueno, com maior teor de lignina total e 
menores teores de holocelulose e cinzas do que as demais espécies.

A madeira de jurema-preta teve três vezes menos cinzas do que a madeira de angico-vermelho e a metade do obtido pela jurema-vermelha.

\section{AGRADECIMENTOS}

Ao Conselho Nacional de Desenvolvimento Científico e Tecnológico (CNPq), pelo apoio financeiro a esta pesquisa.

Aos Professores José Lívio Gomide e Angélica de Cássia Oliveira Carneiro, Universidade Federal de Viçosa, pela colaboração nas análises químicas e anatômicas das madeiras das espécies estudadas, respectivamente.

Aos senhores Pedro Andrade Mota e Sebastião da Silva Dantas, Universidade Federal de Campina Grande, pela valiosa ajuda nos trabalhos de campo e de laboratório, respectivamente.

\section{STATUS DA SUBMISSÃO}

Recebido: $12 / 12 / 2012$

Aceito: 08/07/2013

Publicado: 31/12/2013

\section{AUTOR(ES) PARA CORRESPONDÊNCIA}

\section{Juarez Benigno Paes}

Departamento de Ciências Florestais e da Madeira, Universidade Federal do Espírito

Santo - UFES, Av. Governador Lindemberg, 316, Centro, CEP 29550-000, Jerônimo Monteiro, ES, Brasil

e-mail: jbp2@uol.com.br

\section{REFERENCIAS}

Associação Brasileira de Normas Técnicas - ABNT. NBR 8633: carvão vegetal: determinação do poder calorífico. Rio de Janeiro: ABNT; 1983. 13 p.

Associação Brasileira Técnica de Celulose e Papel - ABTCP. Normas técnicas ABCP. São Paulo: ABTCP, 1974. 18 p.

Burger LM, Richter HG. Anatomia da Madeira. São Paulo: Nobel, 1991. 154 p.
Gomide JL, Demuner BJ. Determinação do teor de lignina em material lenhoso: método Klason modificado. O Papel 1986; 47(8): 36-38.

Instituto Brasileiro de Geografia e Estatística - IBGE. Cidades@ [online]. [cited 2008 jul. 17]. Available from: http://www.ibge.gov.br/cidades@.

Lewin M, Goldstein IS. Wood structure and composition. New York: M. Dekker; 1991. 488 p.

Lima CR, Lima GD, Paes JB. Consumo residencial de energéticos para cocção nas comunidades Santana e Queimadas, Santa Terezinha, Paraíba, Brasil. Biomassa \& Energia 2006; 3(2): 83-96.

Oliveira E. Características anatômicas, químicas e térmicas da madeira de três espécies de maior ocorrência no Semi-Árido Nordestino [tese]. Viçosa: Universidade Federal de Viçosa; 2003. 122 p.

Oliveira E, Vital BR, Pimenta AS, Della Lucia RM, Ladeira AMM, Carneiro ACO. Estrutura anatômica da madeira e qualidade do carvão de Mimosa tenuiflora (Willd.) Poir. Revista Árvore 2006; 30(2): 311-318. http://dx.doi.org/10.1590/S0100-67622006000200018

Oliveira JB, Vivacqua A Fo, Gomes PA. Produção de carvão vegetal: aspectos técnicos. In: Penedo WR, editor. Produção e utilização de carvão vegetal. Belo Horizonte: CETEC, 1982. p. 60-73. (Série de Publicações Técnicas, n. 8).

Panshin AJ, De Zeeuw C. Textbook of technology. 3rd ed. New York: McGraw Hill; 1980. 722 p.

Pettersen RC. Chemical composition of wood. In: Rowell $\mathrm{R}$, editor. The chemistry of solid wood. Washington: American Chemical Society; 1984. p. 54-126. http:// dx.doi.org/10.1021/ba-1984-0207.ch002

Ramalho RS. O uso de macerado no estudo anatômico de madeiras. Viçosa: UFV;1987. 4 p.

Sjöström E. Wood chemistry: fundamentals and applications. 2nd ed. New York: Academic Press; 1993. 293 p.

Steel RGD, Torrie JH. Principles and procedures of statistic: a biometrical approach. 2nd ed. New York: McGraw Hill; 1980. 633 p.

Superintendência de Administração do Meio Ambiente - SUDEMA. Atualização do Diagnóstico Florestal do Estado da Paraíba. João Pessoa: SUDEMA; 2004.268 p

Vital BR. Métodos de determinação da densidade da madeira. Viçosa: SIF; 1984. 21 p. (Boletim Técnico, n. 1).

Xavier EPL, Paes JB, Lira JA Fo . Potencial madeireiro de duas microrregiões do Estado da Paraíba. Biomassa \& Energia 2005; 2(2): 103-112. 